\title{
GEDI AND TANDEM-X FUSION FOR 3D FOREST STRUCTURE PARAMETER RETRIEVAL
}

SeungKuk Lee ${ }^{1,2}$, Lola Fatoyinbo ${ }^{1}$, Wenlu $Q i^{2}$,

Steven Hancock ${ }^{2}$, John Armston ${ }^{2}$, Ralph Dubayah ${ }^{2}$ 


\section{Earth Ventures Instrument (EVI)}

GEDI: Global Ecosystem Dynamics Investigation

- Selected in late 2014 for \$94 M (Class C mission)

- Multi-beam waveform lidar instrument

- NASA Goddard Spaceflight Center (GSFC)

- Deployed on International Space Station

- Launch on SpaceX-17: Nov 2018

- Observations between +/- 50N/S

- Nominal 2 year mission length

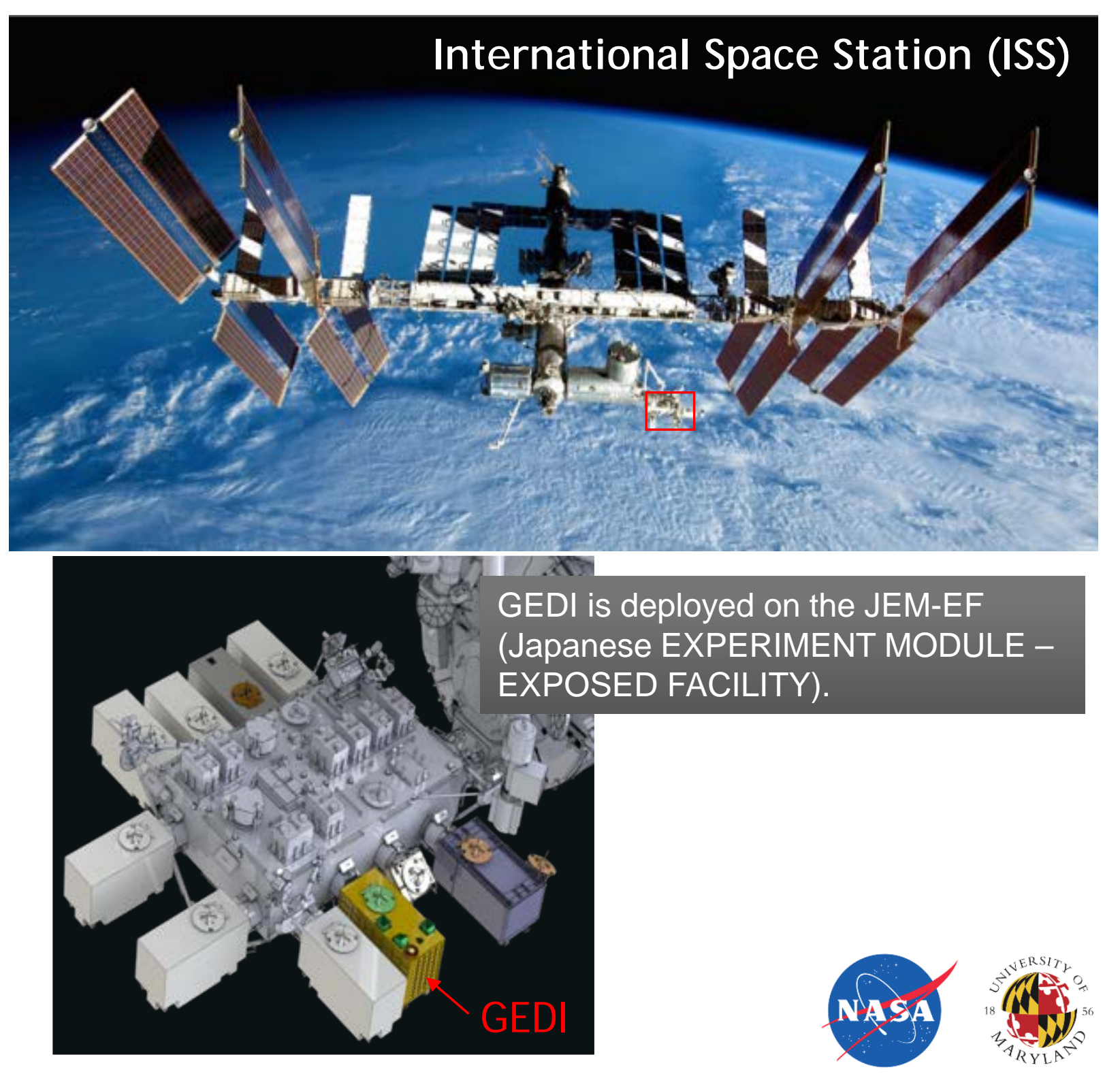




\section{Mission Overview}

\section{High Resolution Laser Ranging of the Earth's Forests and}

- GEDI produces high resolution laser ranging observations of the 3D structure of the Earth.

- GEDI makes precise measurements of forest canopy height, canopy vertical structure, and surface elevation.

- GEDI improves our ability to characterize important carbon and water cycling processes, biodiversity and habitat.

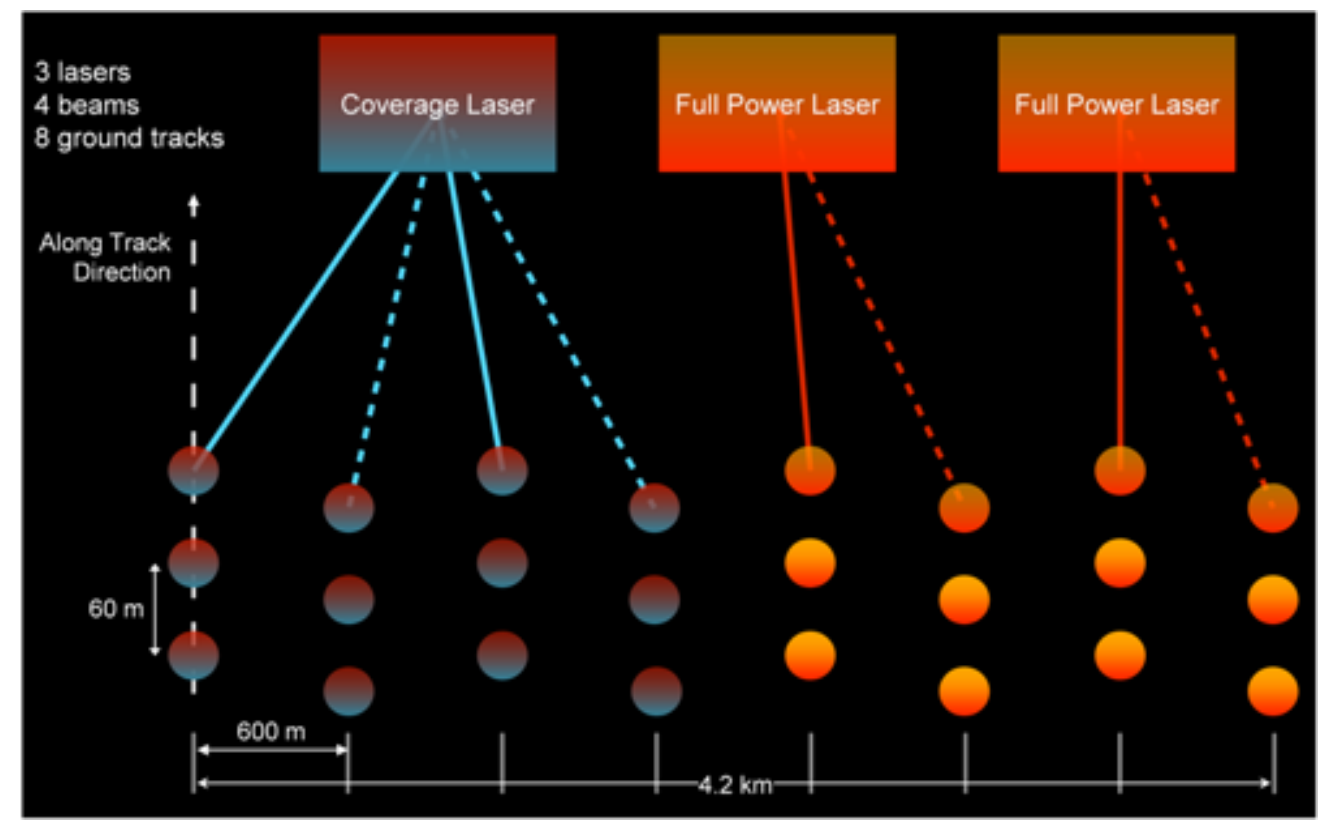

GEDI uses 3 lasers to produce 10 transects of lidar waveforms. 


\section{Science Questions and Objectives}

GEDI Goal: Advance our ability to characterize the effects of changing climate and land use on ecosystem structure and dynamics
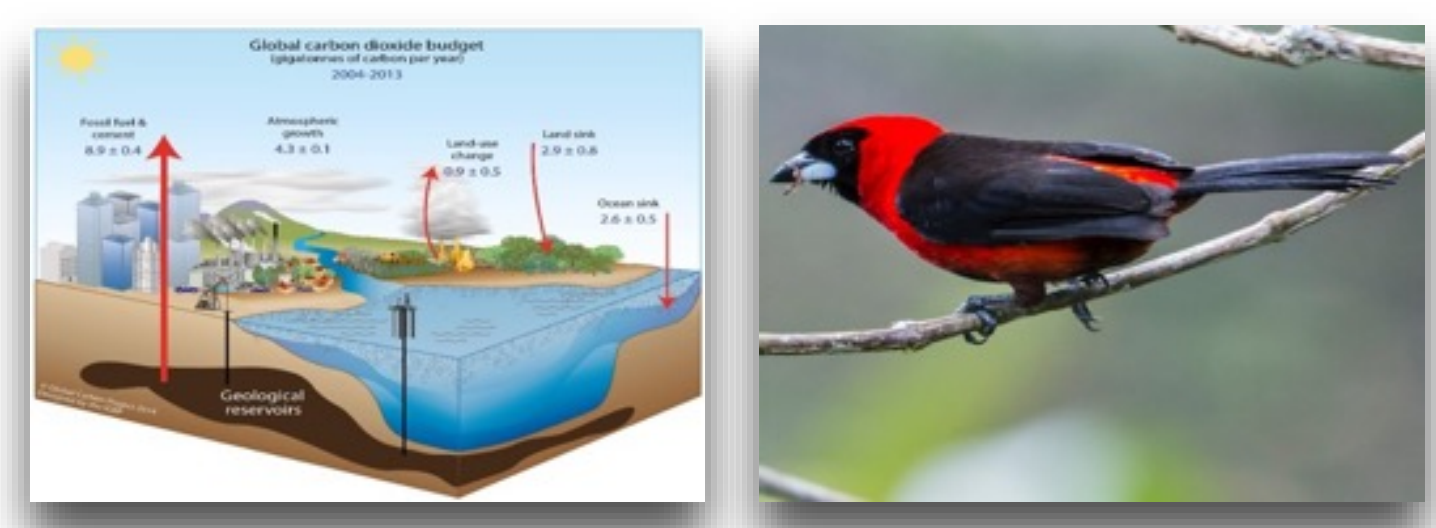

Carbon Cycle

$$
\text { Biodiversity }
$$

\section{Question}

What is the carbon

balance of the

Earth's forests?

How will the land surface mitigate

atmospheric $\mathrm{CO} 2$ in the future?

How does forest structure affect habitat quality and biodiversity?
Quantify

Forest Biomass

Disturbance and Recovery

Carbon

Sequestration Potential

Vertical Forest Structure and its

Relationship to Biodiversity 


\section{GEDI Lidar Measurements}
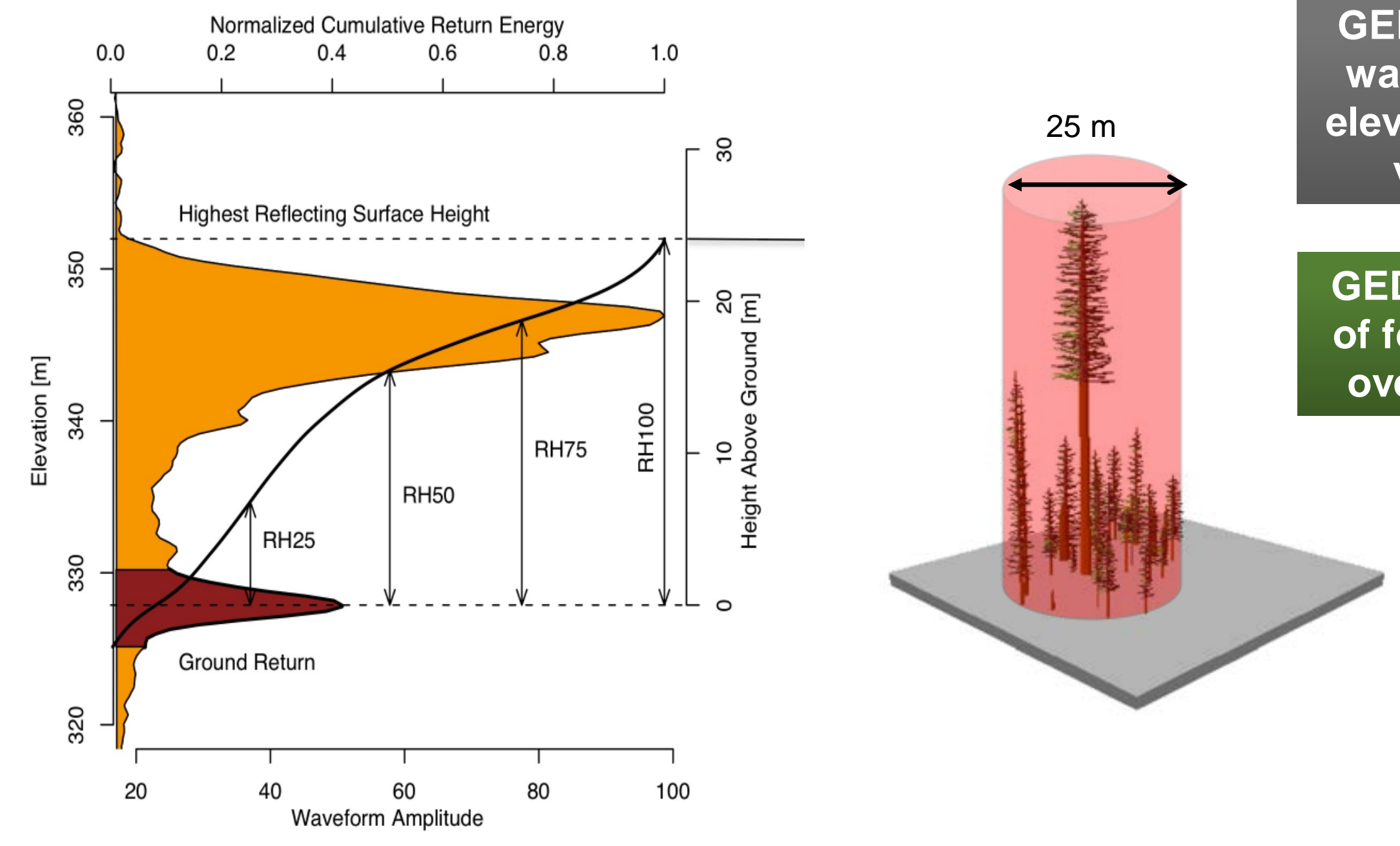

GEDI's sole observable is the lidar waveform which provides ground elevation, canopy height, cover and various profiles and metrics.

GEDI makes 12 billion observations of forest and land surface structure over its nominal two-year mission 


\section{Science Approach and Data Products}

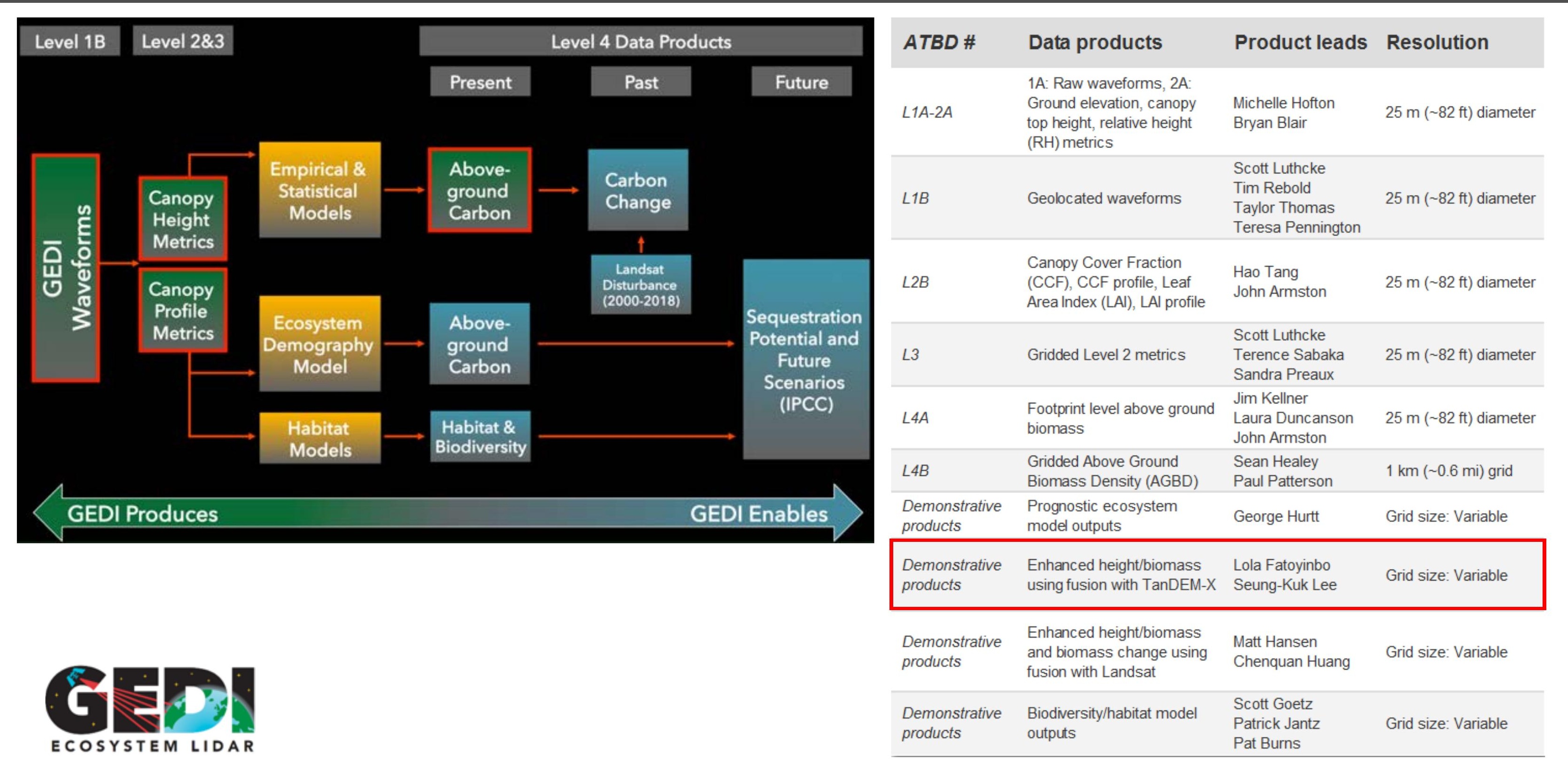




\section{GEDI \& TanDEM-X Fusion}

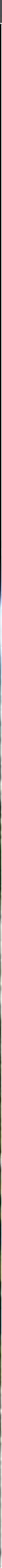




\section{GEDI Data + Single pol. TanDEM-X}

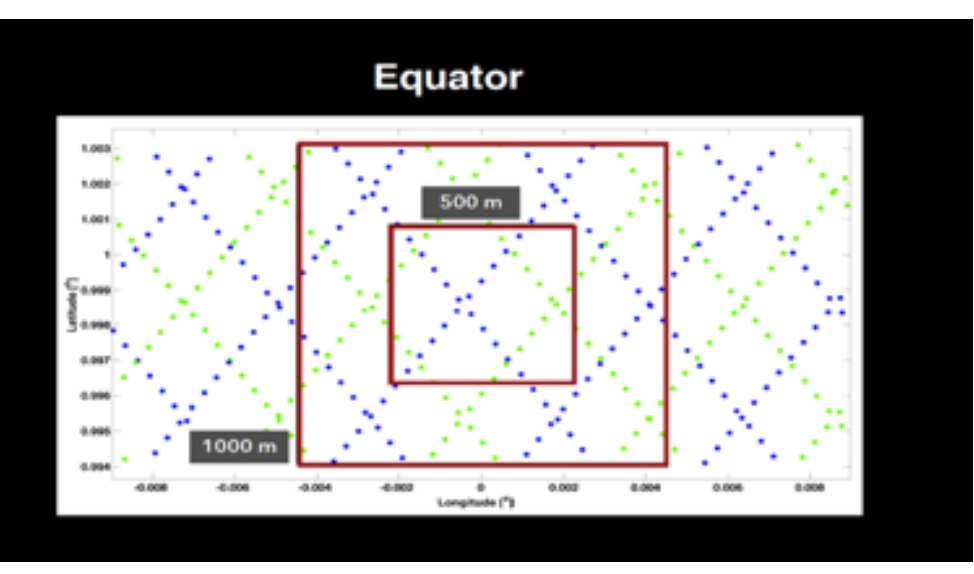

\section{Single-polarization (HH) Pol-InSAR Inversion (RVoG model)}

\section{Polarization}

Single-Pol.

\section{Independent} Coherence

Method1: Extinction $\sigma$

¿Qi et al. Remote Sensing of Environment 2016〉

- Using GEDI RH metrics, volume coherence is simulated on each GEDI footprint. $\rightarrow$ Optimization of the extinction

- Interpolation of $\sigma$ in a grid

\section{Method2: Ground Phase $\phi_{0}$}

- GEDI ground-level DEM on each GEDI footprint $\rightarrow$ Interpolation

- Merging $\rightarrow$ GEDI DTM and TanDEM-X DEM 


\section{Multi-Baseline TDX Inversion; Fusion DTM}

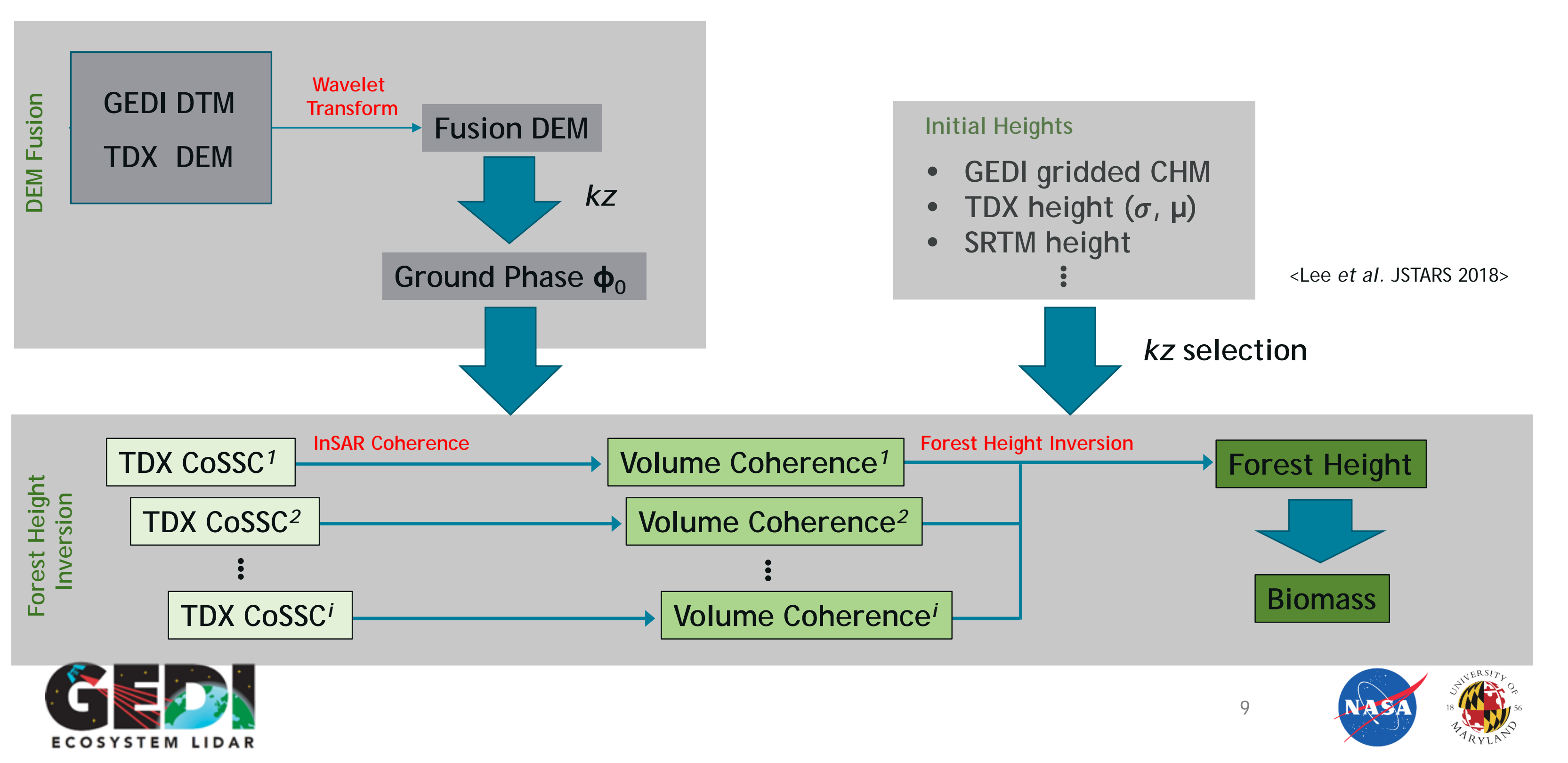




\section{GEDI and TDX DEMs Merging; Wavelet}

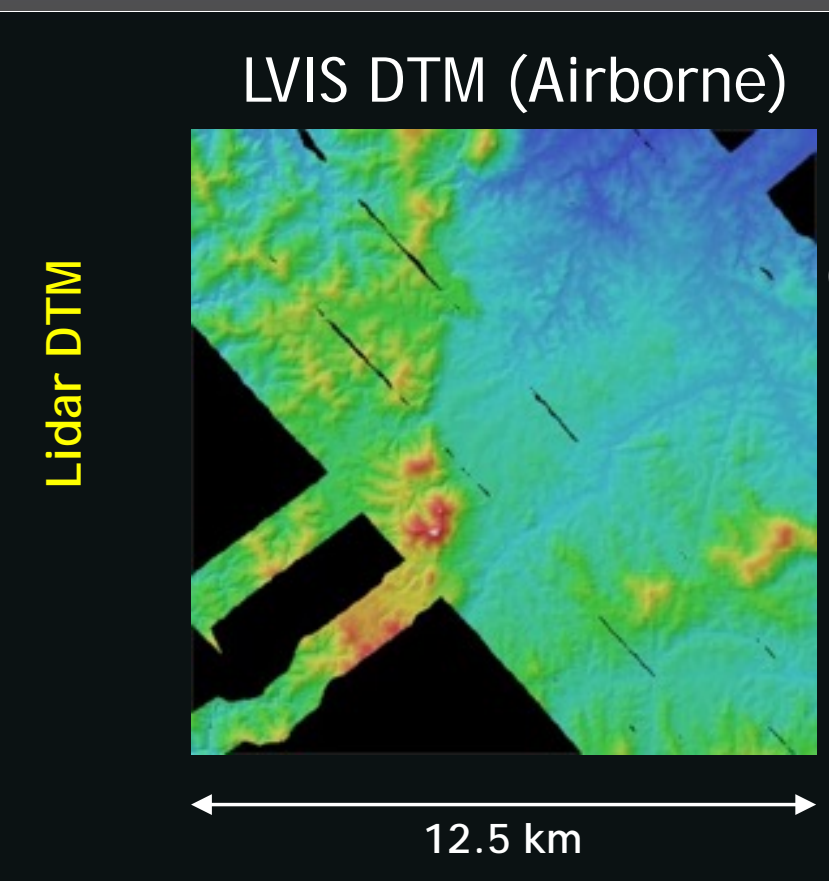





TanDEM-X DEM (Spaceborne)
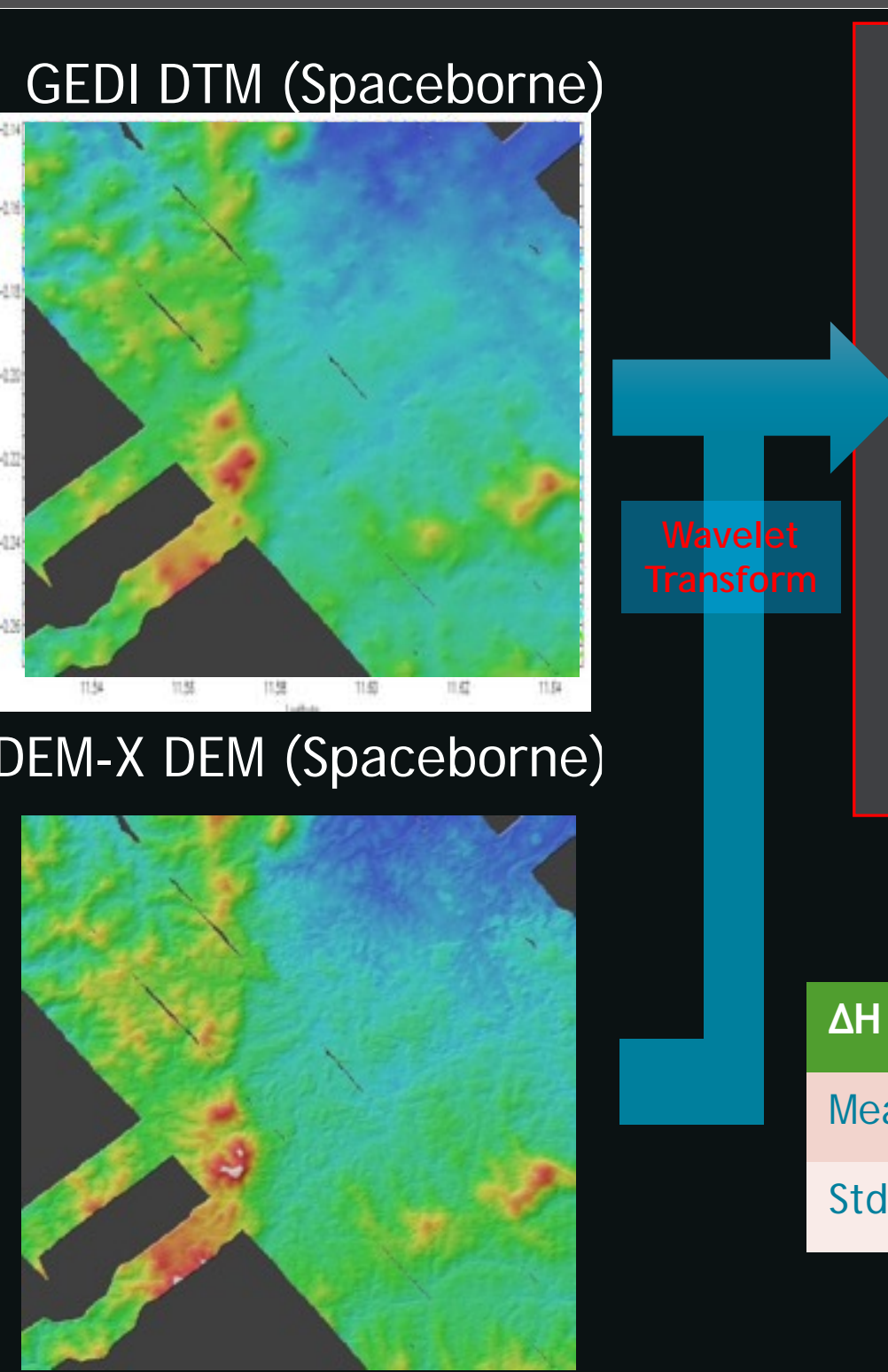

GEDI + TDX DTM (Fusion)

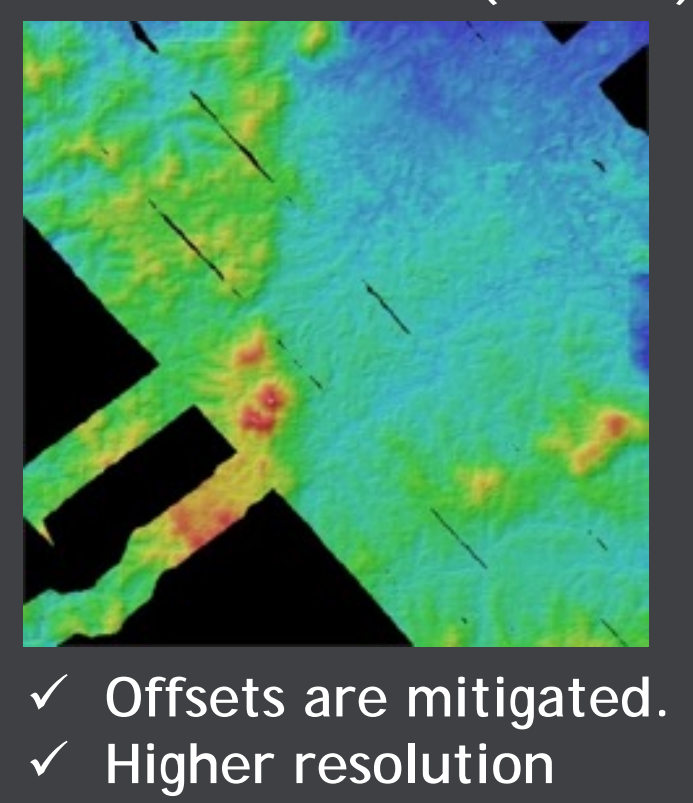

$650 m$

$0 \mathrm{~m}$

\begin{tabular}{lcrc}
\hline $\mathrm{H}$ & Fusion DTM & GEDI DTM & TDX DEM \\
Mean & $0.4 \mathrm{~m}$ & $0.2 \mathrm{~m}$ & $23.1 \mathrm{~m}$ \\
Std. & $8.8 \mathrm{~m}$ & $11.7 \mathrm{~m}$ & $12.4 \mathrm{~m}$
\end{tabular}




\section{Forest Height Inversion Results}

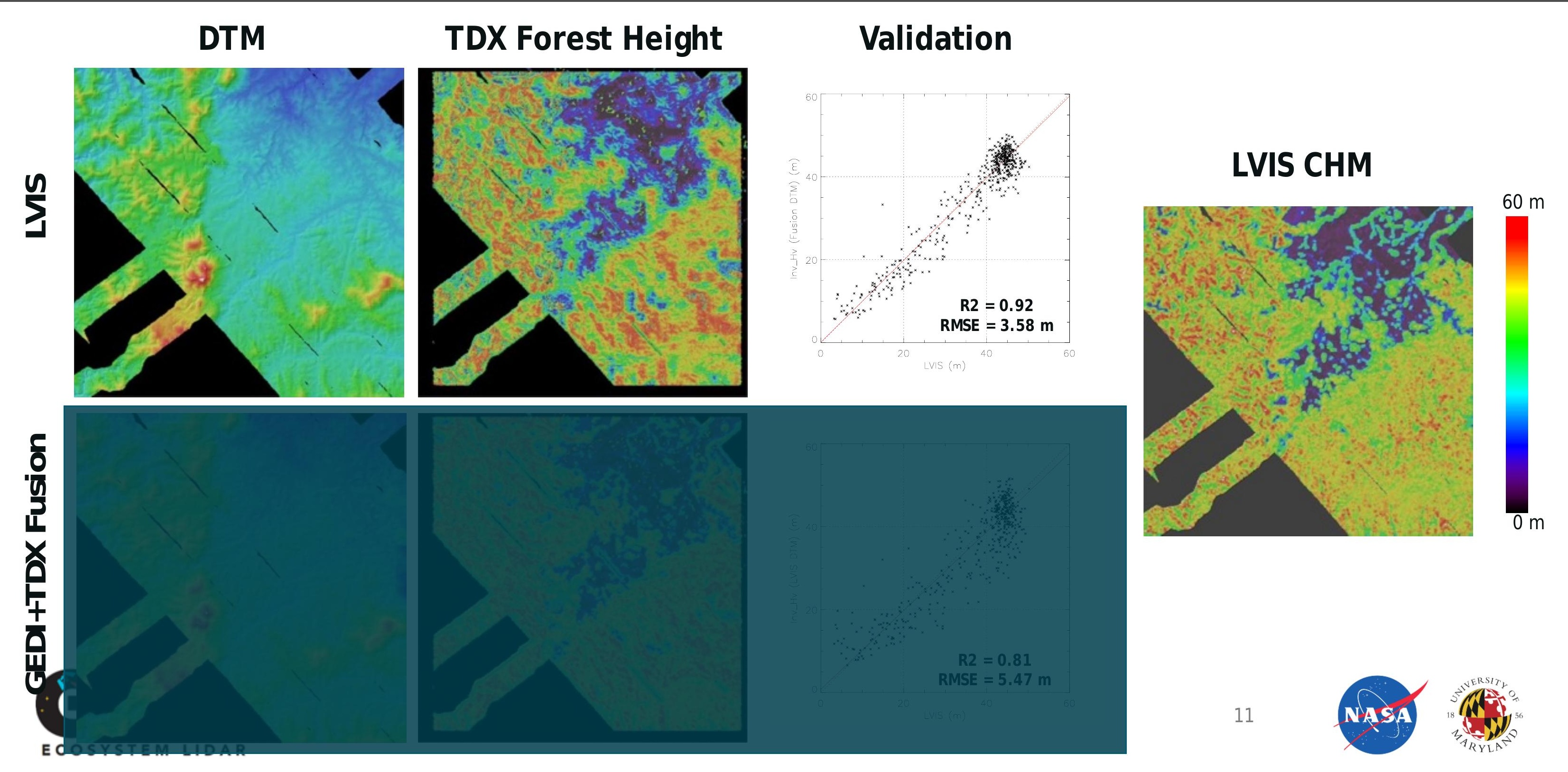




\section{Forest Height Inversion Results}

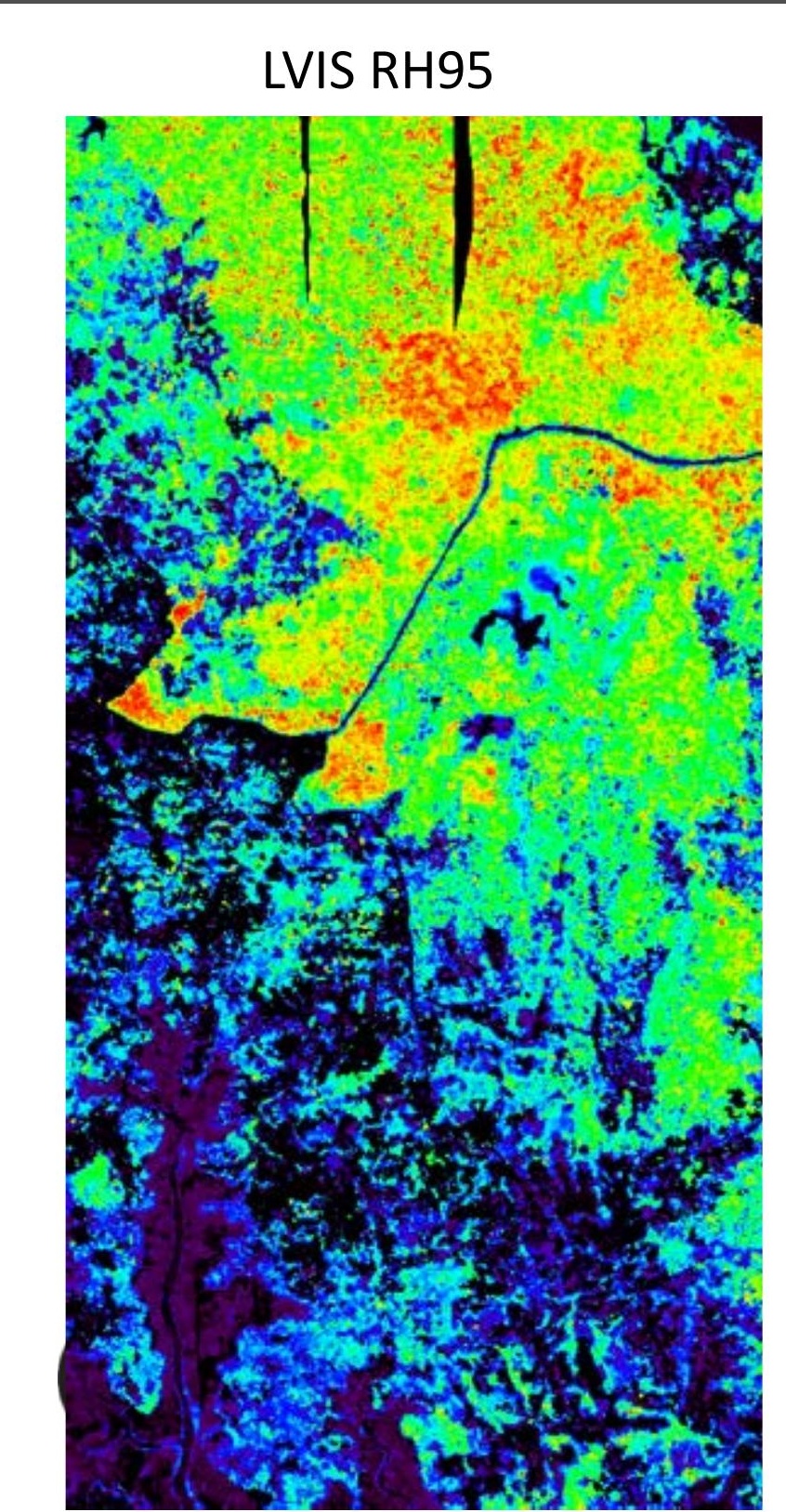

Inversion using LVIS DTM
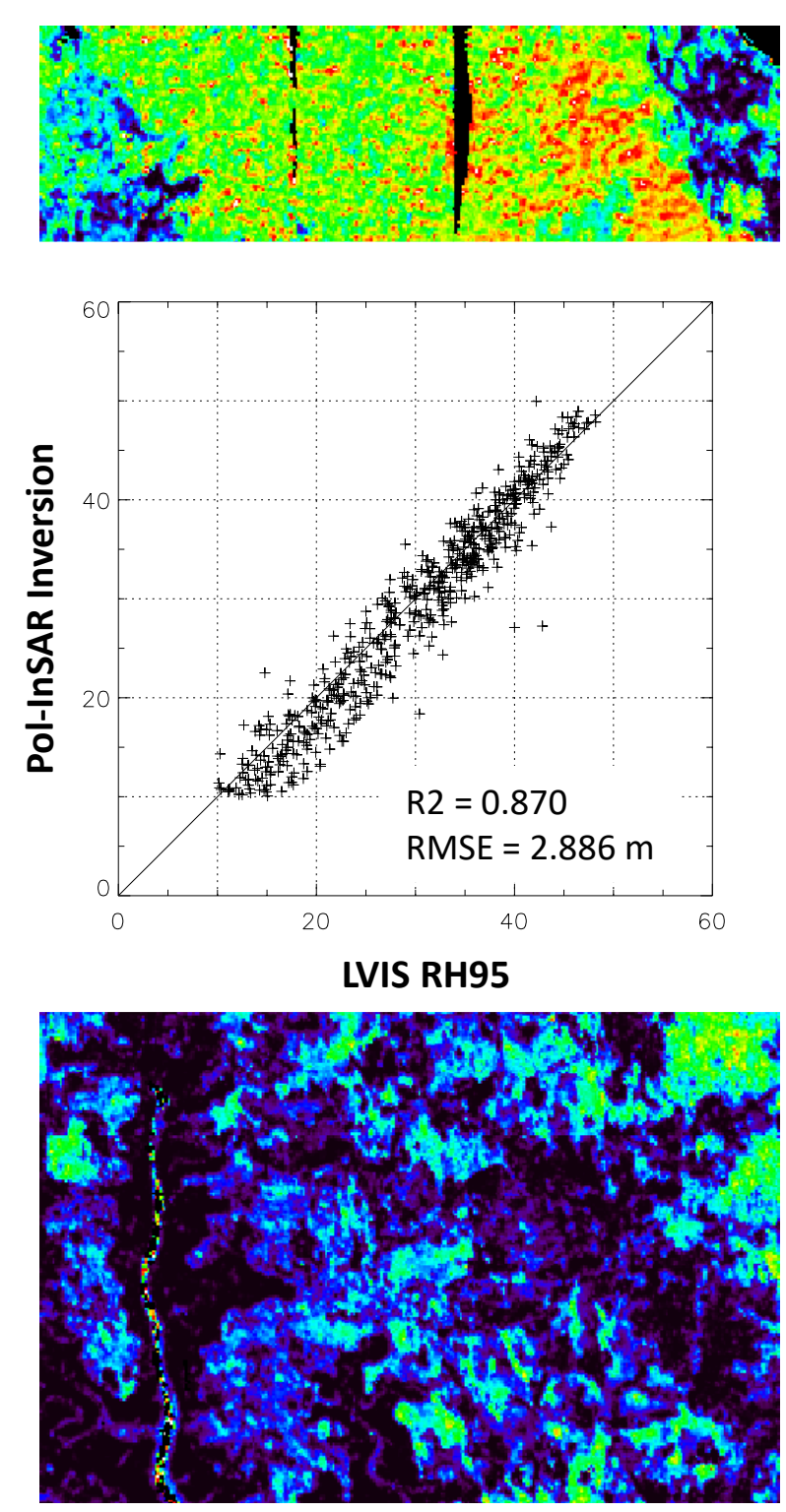

Inversion using Fusion DTM
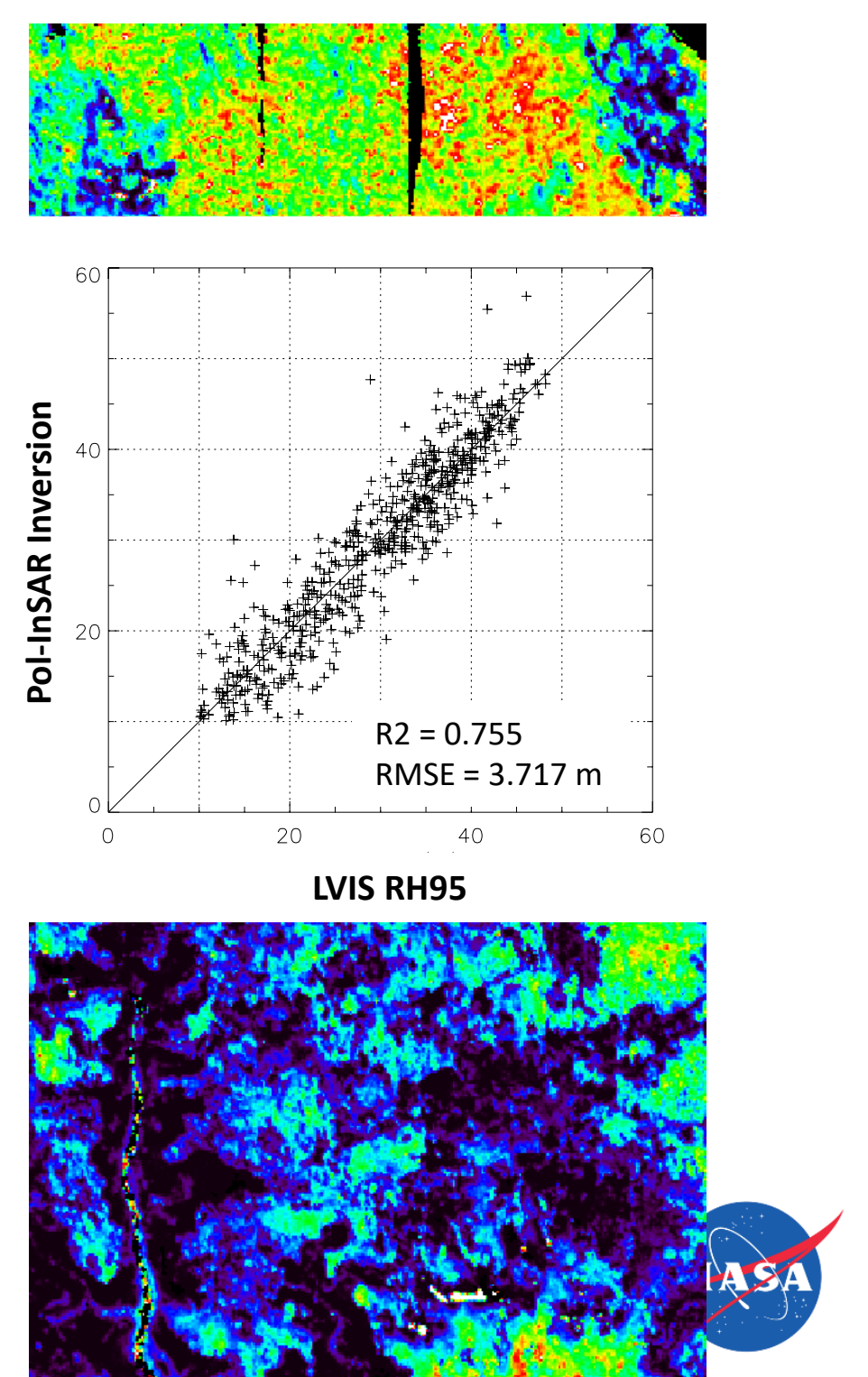

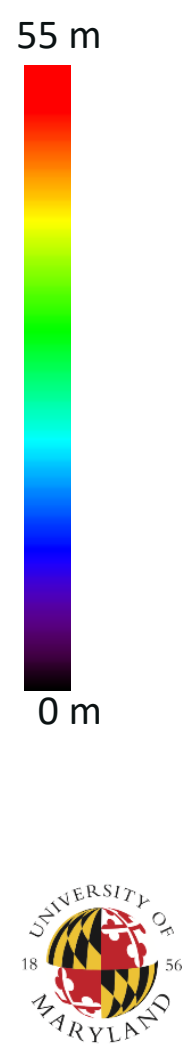




\section{Aboveground Biomass}

\section{Forest Height Map}
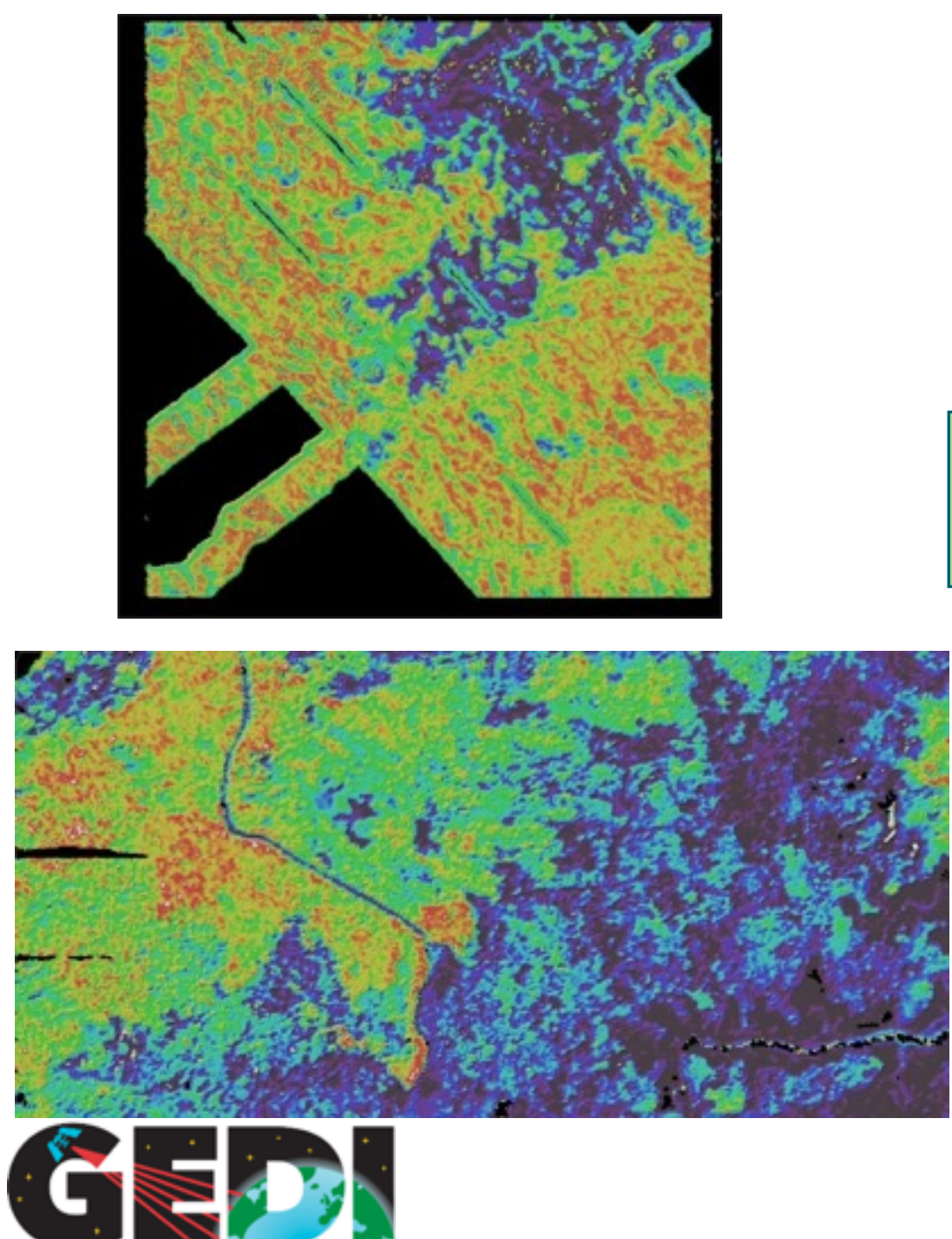

Height-biomass allometric Eq. from GEDI waveform lidar data

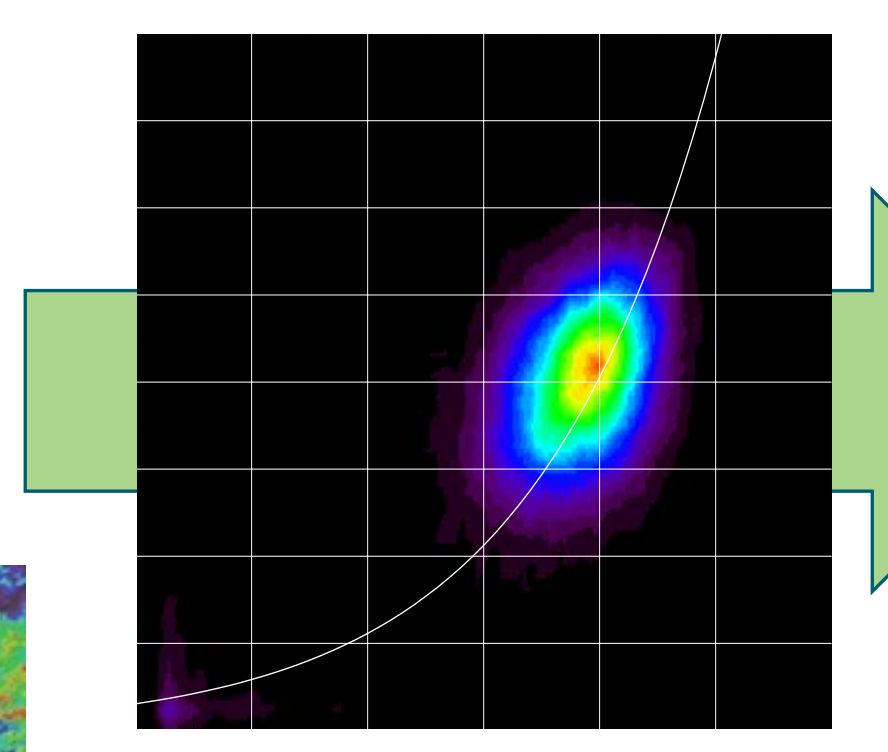

Aboveground Carbon Map
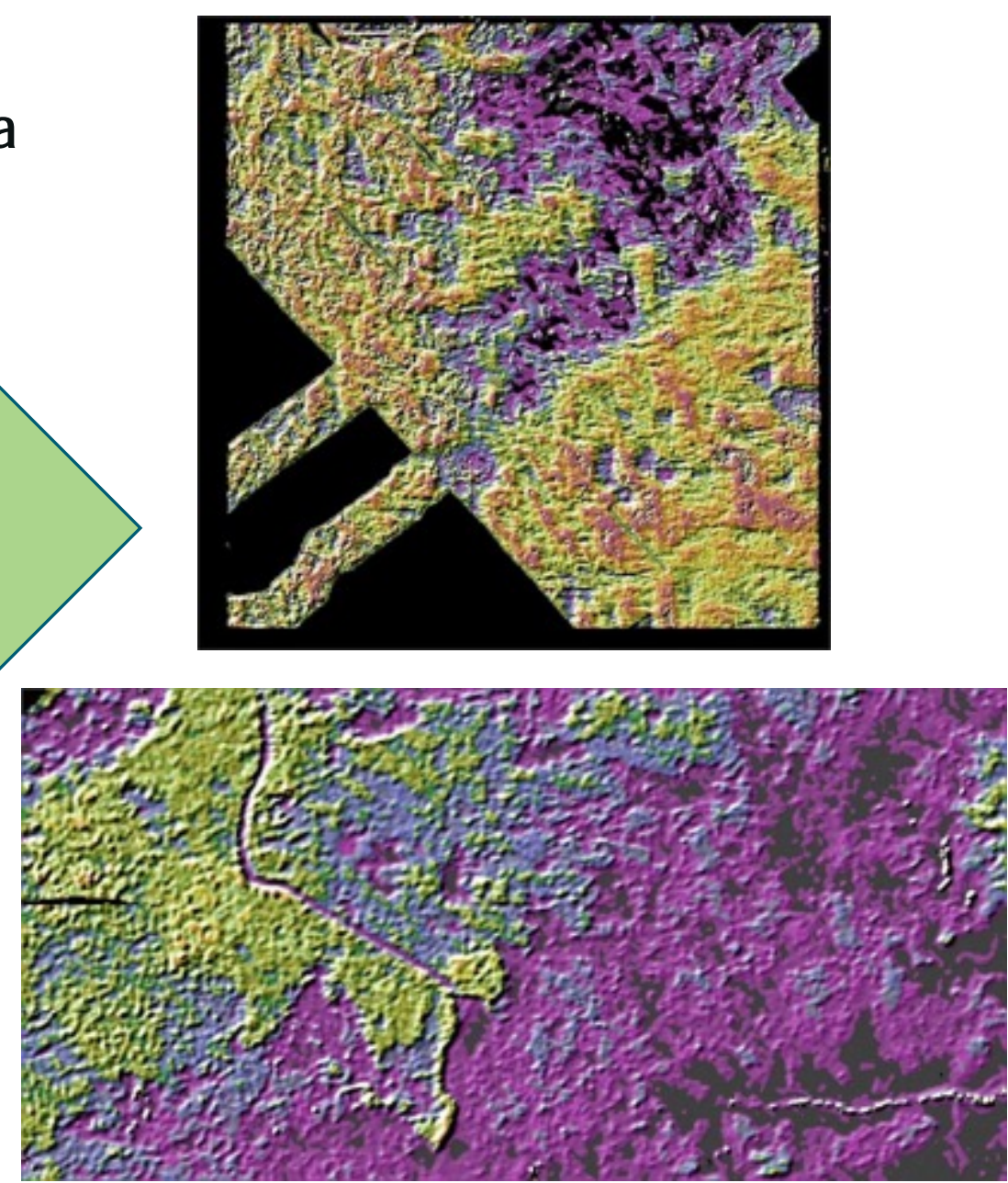


\section{GEDI Webpage: https://gedi.umd.edu}

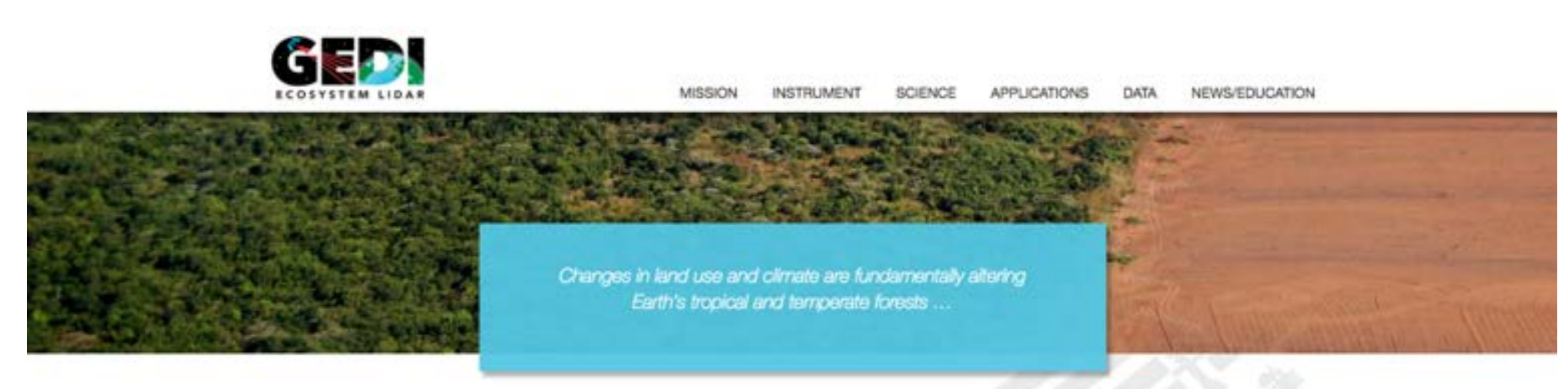

Global Ecosystem Dynamics Investigation (GEDI) High resolution laser ranging of Earth's forests and topography from the International Space Station (ISS)

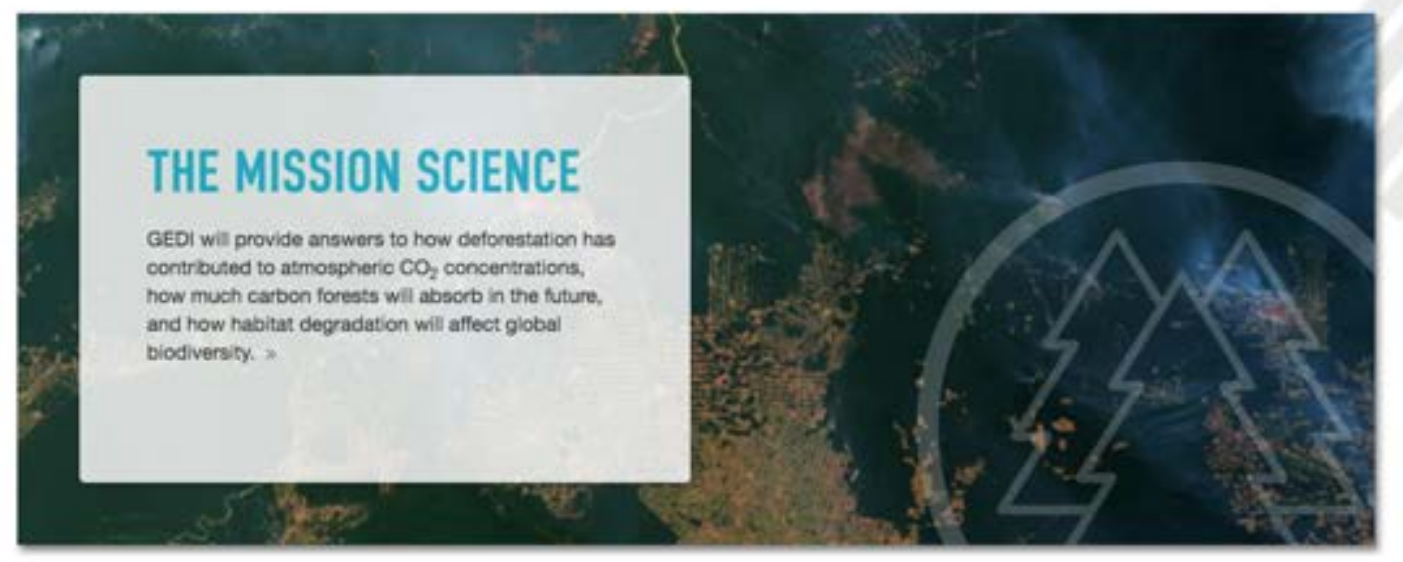


Thank you! 